\title{
Delivering Machine Readable Microlensing Data Across the Internet
}

\section{Ian Bond ${ }^{1}$, Wei Lin, and Winston Sweatman}

Institute for Information and Mathematical Sciences, Massey University, Private Bag 102904, North Shore City 0745, New Zealand

E-mail: $\{$ i.a.bond, w.lin, w.sweatman\} @massey.ac.nz

Real-time microlensing data are delivered across the Internet via human readable emails and displays in a web browser. There are now a number of scenarios where machine-readable access to microlensing data is required. Here we describe an event "publisher" for MOA that will be in place for the 2008 microlensing season. Events will be delivered using XMPP based instant messaging technologies and event metadata will be encoded using the VOEvent standard. The classical channel of sending out emails and displaying data on a web page will still be used.

The Manchester Microlensing Conference: The 12th International Conference and ANGLES

Microlensing Workshop

Manchester, UK

21-25 January, 2008

$1 \quad$ Speaker 


\section{Introduction}

Following the first detections of microlensing events in the early 1990s, the process of observing and studying them has followed pretty much the same model. The initial detection of microlensing events is the responsibility of survey projects that employ large CCD cameras mounted on telescopes with wide fields-of view. The Optical Gravitational Lensing Experiment (OGLE) and the Microlensing Observations in Astrophysics (MOA) collaborations are two such projects, operating today, that provide this service. The Massive Compact Halo Objects (MACHO) collaboration was another microlensing survey project that operated during the 1990s. Following, an initial alert, microlensing events are then observed by "follow-up" telescopes. There are a number of collaborations that operate networks of follow-up telescopes. These include PLANET, MicroFUN, ROBONET, and MONET, together with a few standalone telescopes. Some of these are geared up mainly to observe microlensing events while others have more wide ranging goals in following up astronomical transients. The microlensing survey groups also operate in a follow-up mode on their own and each other's events.

For all of this to work, it is necessary for the microlensing survey groups to communicate new events and update ongoing events to the follow-up groups. Traditionally this has been done via email notifications and displaying the latest data at web sites.

\section{The MOA Alert System}

The MOA Collaboration operates a $1.8 \mathrm{~m}$ telescope with an 80 Mpixel CCD camera at the Mt John Observatory in the South Island of New Zealand. The procedure by which observational images from the MOA survey telescope are processed into public microlensing alert notifications is summarized in the schematic in Fig. 1 and has been described in detail elsewhere ${ }^{1}$. Difference imaging analysis is used to search for new microlensing in the real-time imaging data. Light curves are generated for all objects located on difference images that may possibly be new transients events. The light curves are then fed into a "Microlensing Analysis Toolkit" that is used to select those that clearly correspond to microlensing on the basis of the shape of the light curve, and to reject all spurious detections. The toolkit is a semi-automated process that combines automatic filtering routines with human interaction at the final step where microlensing events are identified by eye. 


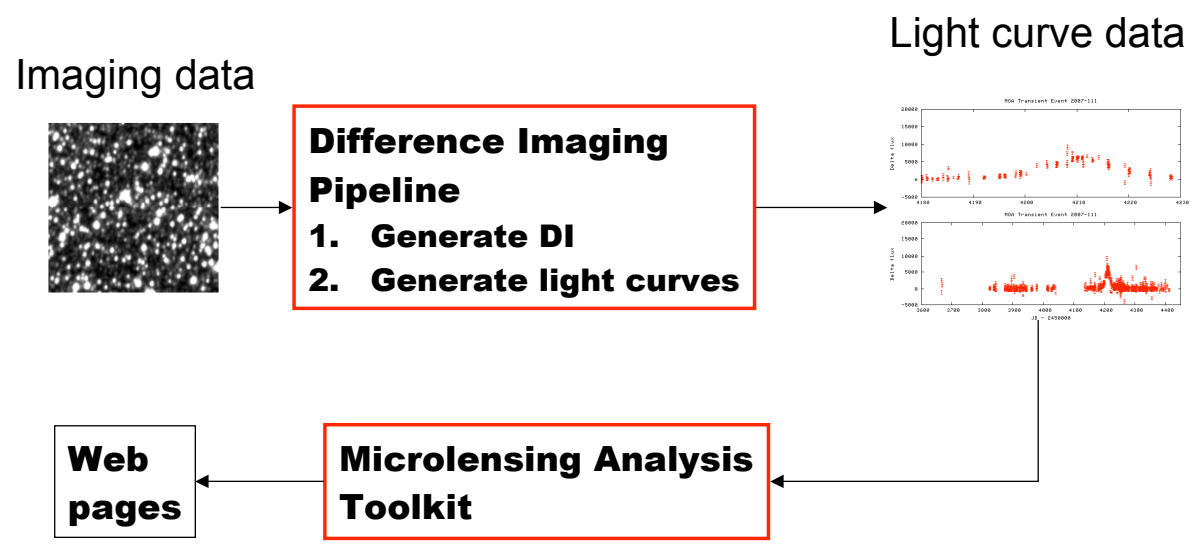

Figure 1. Schematic of the MOA data reduction pipeline showing how the data proceeds from imaging data to microlensing events displayed on a web page.

The current practice followed by both OGLE and MOA (and MACHO before them) is to send out email alerts of any new microlensing events as they are detected, and to display information on new and ongoing events on a web site. Furthermore, prompt email notifications are sent if any event is undergoing some behaviour that requires immediate attention for followup observations. For example, an event may be identified as high magnification or an anomaly may be detected in an ongoing event.

E-mail and web pages are two Internet technologies that are designed for human readability and operation. However, there are a number of scenarios that require machinereadable access to real-time microlensing data. These include:

- Robotic telescope networks that automatically respond to real-time alerts of new events or notifications of urgent observations required for selected ongoing events. These include MONET $^{2}$ and ROBONET ${ }^{3}$. Another architecture that feeds from real-time astronomical data is eSTAR ${ }^{4}$, which employs intelligent agents to automatically schedule observations.

- Specialized software applications that look for microlensing anomalies in real-time feeds of microlensing data. These include SIGNALMEN ${ }^{5}, \mathrm{PLOP}^{6}$, and ARTEMIS ${ }^{7}$.

One common procedure is to write "screen scraper" scripts that download the source files intended for web browsers and parses the HTML source for the required information. Such practices are not satisfactory and really constitute an abuse of the underlying technologies for displaying data in web browsers. The International Virtual Observatory Consortium has devised an XML schema called VOEvent ${ }^{8}$ that is designed to provide machine-readable descriptions of astronomical phenomena that are time-critical in nature. 


\section{Machine Readable MOA Events}

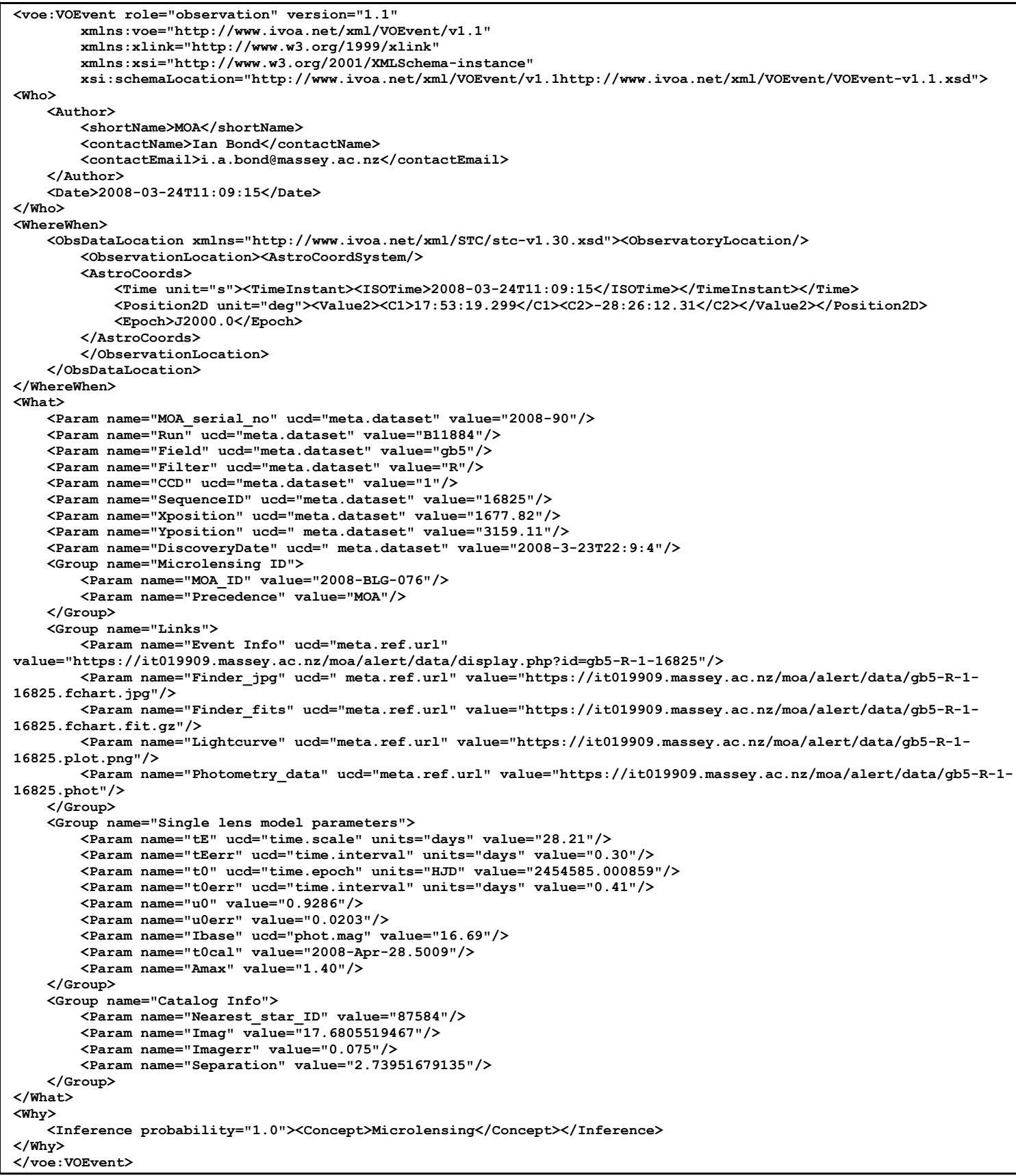

Fig. 2. A machine readable microlensing alert for MOA 2008-BLG-076 encoded using the VOEvent schema. VOEvent divides event metadata into a number of XML stanzas. Here the $\langle$ Who $>$... $</$ Who $>$ stanza specifies contact details for this event together with the date the event was issued; <WhereWhen $>$ specifies the coordinates of the event; <What $>$ specifies various observation specific data including the internal ID information, links to finder charts, data files, and web pages for the event, the results of fitting a single lens model to the event, and information from the MOA catalog of resolved stars; $\langle$ Why $\rangle$ gives a machine readable scientific description of the event where, in this case, it is identified as microlensing with $100 \%$ confidence. 
In developing a system for providing real-time feeds of microlensing data, it is useful first to consider the lifecycle of a microlensing event. One can visualize a microlensing event as comprising a sequence of messages. The first message provides notification that a new microlensing event has been detected. This is then followed by a sequence of "update" messages. An update could include: new photometry available for this ongoing event, new microlensing parameters that have been calculated, a high magnification alert, an anomaly alert, etc.

We have designed an implementation that encodes the various messages in a microlensing event lifetime as data packets that conform to the VOEvent schema. In Fig. 2, we show an example VOEvent packet representing an alert of a newly detected microlensing event. This can be thought of as a machine readable microlensing alert, in contrast to a human readable alert that one sees on a web page or in an email message.

\section{Delivering MOA VOEvent packets}

To deliver MOA VOEvent data across the Internet we have adopted the publish/subscribe extension to the common instant messaging model. The publish/subscribe extension is more convenient that pure instant messaging because it decouples all the messaging users (or clients) from each other. Rather than an alert client having to maintain a list of recipients, the alert client "publishes" a message to a particular "channel" on an instant messaging server. This message is then received by all other users who "subscribe" to that channel. 


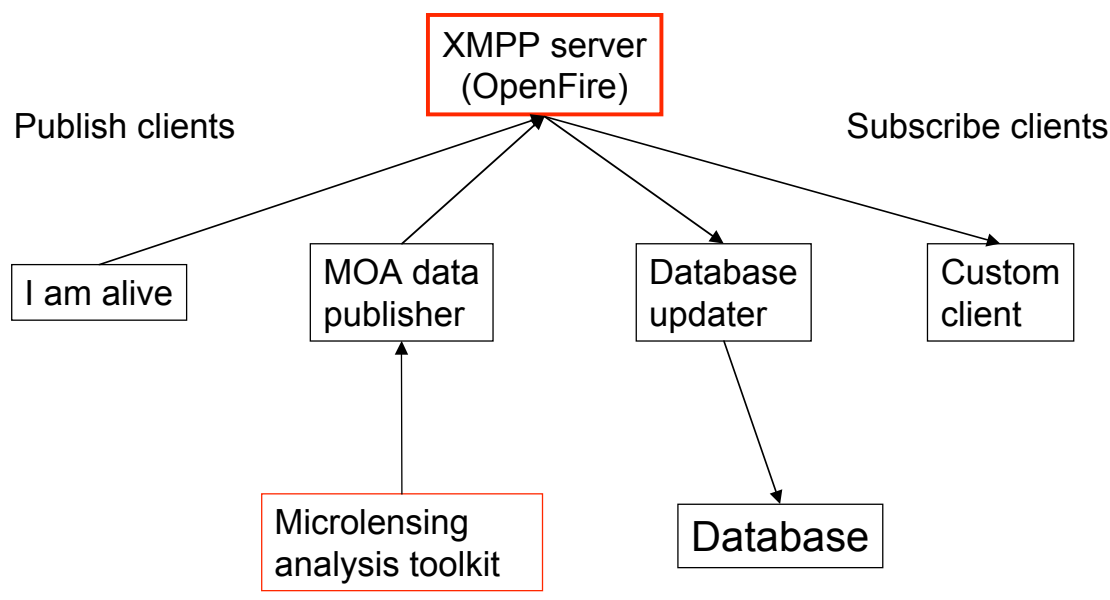

Fig. 3. Publish/subscribe architecture for delivering MOA VOEvents.

Our VOEvent architecture is depicted in Fig. 3. We have adopted the open standards XMPP $^{9}$ (Extensible Messaging and Presence Protocol) for instant messaging and the XEP$0060^{10}$ extension specification for XMPP. We have deployed the OpenFire application server on a host PC at Massey. OpenFire ${ }^{11}$ is an XMPP instant messaging server that supports publish/subscribe and it functions in a way analogous to a webserver. The use of publish/subscribe gives us the flexibility to deploy a number of specialized subscriber clients that perform specific functions. One of these is an email relay program that will automatically send an email message to recipients on a mailing list for newly detected microlensing events. Another subcriber client is a program that updates a database of information on all microlensing events. A series of PHP scripts have also been written that will display this information in a web browser. This is for those who do not require machine readable access to MOA data and simply wish to access the data via a web browser - as has always been the case previously.

VOEvents are delivered as the message payload of an XMPP formatted message. These can be accessed via the TCP port 5222. Anyone can write their own application software to directly access the MOA VOEvent data feeds. In order to do this, an API that supports XMPP and publish/subscribe XEP-0060 extensions is required. For Java programmers, one can use the Smack $^{12}$ library for XMPP support together with the Su-Smack ${ }^{13}$ extensions for XEP-0060.

The MOA VOEvent alert system is now operational as of 2008. Currently our set-up sends out VOEvent packets only for those events that are clearly microlensing. We plan to expand this in future to include all astronomical transients events. These include cataclysmic variables, flare stars, and asteroids that also show up in the MOA survey. The VOEvent schema has a <Why $>$ stanza that can be used to provide a scientific assessment of the event by assigning "inference" weightings to the different possibilities. 


\section{References}

[1] I.A. Bond et al., Real-time difference imaging of MOA Galactic Bulge observations during 2000, MNRAS 327, 868, (2001)

[2] F.V. Hessman and K. Beuermann, MONET: Monitoring Network of Telescopes, The Physics of Cataclysmic Variables and Related Objects, ASP Conference Proceedings, Vol. 261. Edited by B. T. Gänsicke, K. Beuermann, and K. Reinsch. ISBN: 1-58381-101-X. San Francisco: Astronomical Society of the Pacific, 2002, p. 674.

[3] S.N. Fraser, Scheduling for Robonet-1 homogenous telescope network, Astron. Nachr., .327, 779 (2006).

[4] A. Allan, The eSTAR network - agent architectures for astronomy, Astron. Nachr., 327, 767 (2006).

[5] M. Dominik et al., An anomaly detector with immediate feedback to hunt for planets of Earth mass and below by microlensing, MNRAS, 380, 792 (2007).

[6] K. Horne, these proceedings.

[7] ARTEMiS (Automated Robotic Terrestrial Exoplanet Microlensing Search): A possible expertsystem based cooperative effort to hunt for planets of Earth mass and below, Astron. Nachr., 329, 248 (2008).

[8] R. Seaman et al., Sky Event Reporting Metadata (VOEvent)_Version 1.11, available at: http://ivoa.net/Documents/latest/VOEvent.html, November 1, 2006

[9] http://www.xmpp.org/, accessed April 14, 2008.

[10] P. Millard, P. Saint-Andre, \& R. Meijer, XEP-0060: Publish-Subscribe, http://www.xmpp.org/extensions/xep-0060.html, March 3, 2008.

[11] OpenFire available from: http://www.igniterealtime.org/projects/openfire/index.jsp, accessed April 14,2008

[12] Smack documentation: http://www.igniterealtime.org/builds/smack/docs/latest/documentation/, accessed April 14, 2008.

[13] Su-smack documentation: http://static.devel.it.su.se/su-smack/apidocs/index.html, accessed April $14,2008$. 